\title{
MORSE INDEX OF A CYCLIC POLYGON. II
}

\author{
A. ZHUKOVA
}

\begin{abstract}
A polygonal linkage can be imagined as a set of $n$ rigid bars connected by links cyclically. This construction lies on a plane and can rotate freely around the links, with allowed self-intersections. On the moduli space of the polygonal linkage, the signed area function $A$ is defined. G. Panina and G. Khimshiashvili proved that cyclic configurations of a polygonal linkage are the critical points of $A$. Later, G. Panina and the author described a way to compute the Morse index of a cyclic configuration of a polygonal linkage. In this paper a simple formula for the Morse index of a cyclic configuration is given. Also, a description is obtained for all possible local extrema of $A$.
\end{abstract}

\section{§1. INTRODUCTION}

We study polygonal linkages with two fixed vertices and their flexes on the plane with allowed self-intersections. Informally, a polygonal linkage can be imagined as $n$ rigid bars on the plane connected by links, cyclically. This mechanism can freely rotate in the links and the bars may cross each other.

The precise definitions are as follows.

A polygonal linkage is a set of $n$ positive numbers $l_{1}, l_{2}, \ldots, l_{n}(n>2)$ that can be realized as the set of lengths of some polygon's sides.

A configuration of a polygonal linkage is a set $P=\left(p_{1}, p_{2}, \ldots, p_{n}\right)$ of $n$ points on the plane such that $\left|p_{i}, p_{i+1}\right|=l_{i}$ (we put $p_{n+1}=p_{1}$ ). The first two points are fixed: $p_{1}=(0,0)$ and $p_{2}=\left(l_{1}, 0\right)$.

The points $p_{i}$ are called vertices of the configuration, and the oriented segments $\overrightarrow{p_{i} p_{i+1}}$ connecting them are called edges. All edges of the configuration $P$ make an oriented polyline, which will be identified with $P$. On pictures, the orientation will be marked with arrows.

The set of configurations of a polygonal linkage with two fixed vertices $p_{1}$ and $p_{2}$ is a moduli space of the polygonal linkage (it can be viewed as a subspace of the Euclidean space $\left.\mathbb{R}^{2 n-4}\right)$. In general, the moduli space is a smooth manifold, with dimension equal to $(n-3)$ (see [3]). We denote it by $\mathcal{M}(L)$.

The moduli space $\mathcal{M}(L)$ is a well-studied object. It depends on the lengths of the sides $l_{i}$. For $n=5,6$ it was explicitly described by Zvonkine in [11]. In [3], Farber and Shuetz computed homology groups of $\mathcal{M}(L)$ for arbitrary number of sides.

On $\mathcal{M}(L)$ we define the signed area function $A(P)$, see Definition 2.1. For a generic polygonal linkage, this function is a Morse function on $\mathcal{M}(L)$. The signed area appears in literature as a natural generalization of area for self-intersecting objects.

A configuration $P$ is said to be cyclic if all its vertices lie on one and the same circle.

2010 Mathematics Subject Classification. Primary 14M06. Secondary 53D30, 58E05.

Key words and phrases. Linkages, moduli space, Morse theory.

Partially supported by the program "Research in Pairs" of Mathematisches Forschungsinstitut Oberwolfach in 2010. The author thanks G. Panina, G. Khimshiashvili, and D. Siersma for their help and useful remarks. 

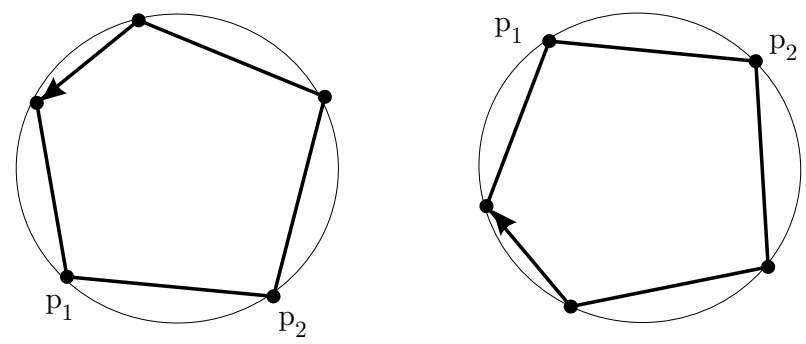

Figure 1. Maximum and minimum of the function $A$.

It has been known for a long time (see the proof of J. Steuner in [9]) that $A$ attains its maximum at a convex, cyclic, positively (i.e., counterclockwise) oriented configuration of the linkage $L$. Therefore, $A$ attains its minimum at an anticonvex (convex and negatively oriented) cyclic configuration of the linkage $L$. The anticonvex configuration is symmetric to the convex configuration with respect to the line $\left(p_{1} p_{2}\right)$ (Figure 1). In [7, Panina and Khimshiashvili proved the following theorem.

Theorem 1.1. Let $L$ be a generic polygonal linkage. Its configuration $P$ is a critical point of the function $A$ if and only if $P$ is a cyclic configuration.

The next natural step is to count the Morse index $m(P)$ of an arbitrary cyclic configuration $P$. In $[8$, a method to count it was suggested. We shall describe this method briefly in $\S 2$.

The main results of the paper are as follows.

The way to count the Morse index, found in [8], is simplified. In Theorem 3.1 we present a simple formula for the Morse index of a cyclic configuration.

In Theorem 4.1 we give a full description of all possible maxima of the function $A$, and its Corollary 4.6 contains a full description of all possible minima of $A$.

\section{$\S 2$. BASIC NOTIONS}

Definition 2.1. Let $P$ be a configuration of a polygonal linkage $L$ with vertices $p_{i}=$ $\left(x_{i}, y_{i}\right), i=1, \ldots, n$. Its signed area is computed in the following way:

$$
2 A(P)=\left(x_{1} y_{2}-x_{2} y_{1}\right)+\cdots+\left(x_{n} y_{1}-x_{1} y_{n}\right) .
$$

We shall use the additivity property for the signed area.

Definition 2.2. Suppose that two configurations $P_{1}=\left(p_{1}, \ldots, p_{n}\right)$ and $P_{2}=\left(q_{1}, \ldots, q_{m}\right)$ of polygonal linkages $L_{1}$ and $L_{2}$ have a common edge with different orientations, i.e., for some $k$ and $l$ such that $1 \leq k \leq n$ and $1 \leq l \leq m$ we have $p_{k}=q_{l+1}$ and $p_{k+1}=q_{l}$. Then the homological sum of $P_{1}$ and $P_{2}$ is defined as the following configuration of some polygonal linkage (see Figure 2):

$$
P_{1}+P_{2}=\left(p_{1}, p_{2}, \ldots, p_{k}, q_{l+2}, \ldots, q_{m}, q_{1}, \ldots, q_{l}, p_{k+2}, \ldots, p_{n}\right) .
$$

In the same way we define the homological sum of configurations with several common edges.

Lemma 2.3 ([7]). Suppose that two configurations $P_{1}$ and $P_{2}$ of polygonal linkages $L_{1}$ and $L_{2}$ have a common edge with different orientation. Then

$$
A\left(P_{1}+P_{2}\right)=A\left(P_{1}\right)+A\left(P_{2}\right) .
$$




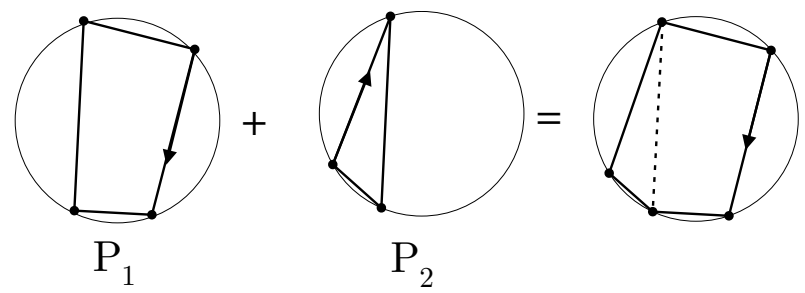

Figure 2. Homological sum of two configurations.

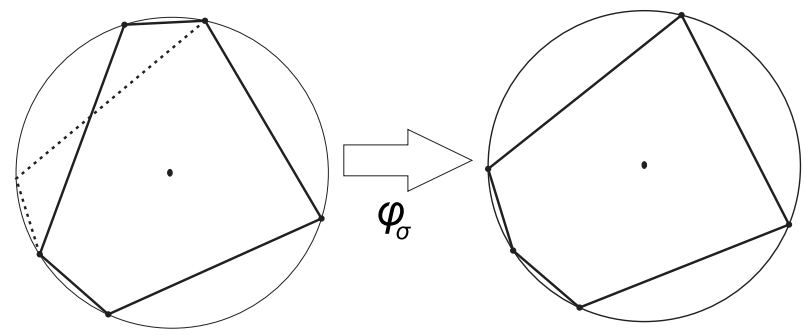

Figure $3 . P$ and $P^{\prime}$ differ by a permutation.

If two polygonal linkages $L_{1}$ and $L_{2}$ differ by a permutation of edges, i.e., $L_{1}=\sigma L_{2}$, where $\sigma$ is an element of the symmetric group $S_{n}$, then there is a natural bijection $\varphi_{\sigma}$ between the critical points of the signed area of $L_{1}$ and $L_{2}$. It can be described as follows. Let $P$ be a cyclic configuration of a polygonal linkage $L$, and let $\sigma \in S_{n}$ be a permutation of edges. To get $\varphi_{\sigma}(P)$, we take a circle of the same radius and "lay out" the edges of $L$ on it in the required order and with the same orientation as in $P$. Since the central angles $\alpha_{i}$ are equal to the corresponding angles in $P$, we get a closed inscribed polyline. This is $\varphi_{\sigma}(P)$ (see, e.g., Figure 3).

Lemma $2.4\left([8)\right.$. The bijection $\varphi_{\sigma}$ does not change the Morse index $m(P)$, the value $A(P)$, and the number $e(P)$ of positively oriented edges.

Definition 2.5. Let $M$ be a subset of the set of vertices of a configuration $P$. Connecting these vertices by edges cyclically in accordance with their order in $P$, we get a cyclic configuration of some polygonal linkage. We call it a subconfiguration of the configuration $P$ corresponding to subset $M$ (see Figure 4).

The following notation will be used.

Let $P=\left(p_{1}, p_{2}, \ldots, p_{n}\right)$ be a cyclic configuration of a polygonal linkage. Then:

$O$ is the center of the circumscribed circle;

$\omega(P)$ is the winding number of $P$ with respect to the center $O$;

$H(P)$ is the determinant of the Hessian matrix of the function $A$ at the point $P$, computed in some coordinate system. $\mathcal{H}(P)=\operatorname{sgn}(H(P))$;

A configuration $P$ is a Morse point for $A$ if $H(P) \neq 0$;

$m(P)$ is the Morse index of the function $A$ at the point $\mathrm{P}$, that is, $m(P)$ is the number of negative eigenvalues of the Hessian matrix of the function $A$ at the point $P$;

$m(P)$ does not depend on the coordinate system chosen, moreover, $(-1)^{m(P)}=\mathcal{H}(P)$;

$\alpha_{i}$ is half of the angle between the vectors $\overrightarrow{O p_{i}}$ and $\overrightarrow{O p_{i+1}}$ (the angle is defined to be positive, orientation is not involved).

A cyclic configuration is said to be central if one of its edges passes through $O$. 
For a noncentral configuration, let $\varepsilon_{i}$ be the orientation of the edge $p_{i} p_{i+1}$, that is,

$$
\varepsilon_{i}= \begin{cases}1 & \text { if the triangle }\left(p_{i}, p_{i+1}, O\right) \text { is positively oriented } \\ -1 & \text { if the triangle }\left(p_{i}, p_{i+1}, O\right) \text { is negatively oriented }\end{cases}
$$

If $\varepsilon_{i}=1$, then we say that the edge $\left(p_{i}, p_{i+1}\right)$ is positively oriented, and if $\varepsilon_{i}=-1$, then the edge $\left(p_{i}, p_{i+1}\right)$ is negatively oriented;

$e(P)$ is the number of positively oriented edges.

Note that any cyclic configuration $P$ of a polygonal linkage $L$ is fully determined by the radius of the circumscribed circle and the orientations of the edges $\varepsilon_{i}, i=1,2, \ldots, n$.

The next metric characteristic will be useful for us.

Definition 2.6. For a noncentral cyclic configuration $P$, put

$$
\delta(P)=\sum_{i=1}^{n} \varepsilon_{i} \tan \alpha_{i}
$$

The main results of the paper are given for generic polygonal linkages. This means that the following conditions are fulfilled.

(1) The moduli space is smooth. The linkages with nonsmooth moduli spaces are described by nonzero linear equations in the parameter space $\mathbb{R}_{+}^{n}$ (see [3]).

(2) All cyclic configurations are noncentral.

(3) All critical points are nondegenerate. The linkages with degenerate critical points are described by a nonzero analytic equation in the parameter space $\mathbb{R}_{+}^{n}$. This equation can be obtained from [8, Theorem 3.1].

(4) No cyclic configuration $P$ satisfies $\delta(P)=0$. The linkages that admit configurations with $\delta(P)=0$ are described with a finite set of nonzero analytic equations in the parameter space $\mathbb{R}_{+}^{n}$. In $[8$, Proposition 4.1] it was proved that they determine a closed set with empty interior.

(5) No cyclic configuration has coinciding vertices.

(6) All conditions 1-5 are fulfilled for the polygonal linkages that correspond to all subconfigurations of all cyclic configurations of $L$.

From the set of all polygonal linkages, each of the above conditions "cuts out" a closed set with empty interior. Taken together, they also "cut out" a closed set with empty interior. Therefore, the set of "generic" polygonal linkages as defined above is open and dense in the set of all polygonal linkages.

Theorem $2.7([8])$. Let $P$ be a generic cyclic configuration. Then

$$
\operatorname{sgn}(H(P))=-\operatorname{sgn}(\delta(P))(-1)^{e(P)} .
$$

Theorem $2.8([8])$. Let $P=\left(p_{1}, \ldots, p_{n}\right)$ be a generic cyclic configuration. Consider its subconfigurations $P_{3}, \ldots, P_{n}$ (see Figure 4$)$ :

$$
P_{i}=\left(p_{1}, \ldots, p_{i}\right), \quad i=3, \ldots, n .
$$

Then the Morse index $m(P)$ is equal to the number of sign changes in the sequence

$$
\mathcal{H}\left(P_{3}\right), \mathcal{H}\left(P_{4}\right), \mathcal{H}\left(P_{5}\right), \ldots, \mathcal{H}\left(P_{n}\right),
$$

where the $\mathcal{H}\left(P_{i}\right)$ for $i=4, \ldots, n$ can be found by Theorem 2.7 , and we put $\mathcal{H}\left(P_{3}\right)=1$.

We shall use the following example.

Example 2.9. There are two types of quadrilateral polygonal linkages: 


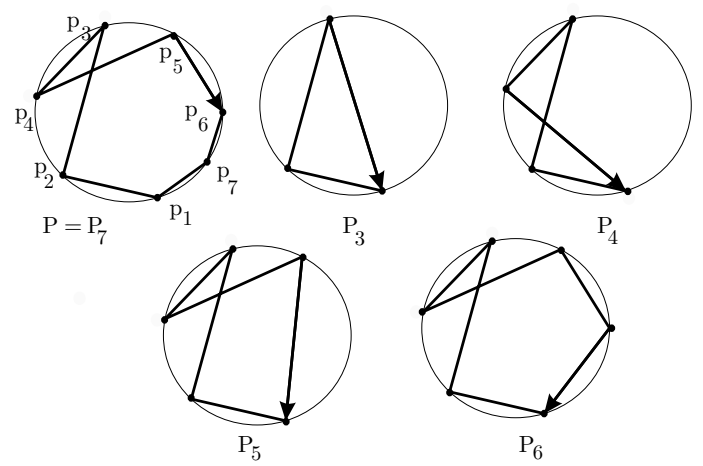

Figure 4. Subconfigurations $P_{3}, \ldots, P_{n}$.

1)

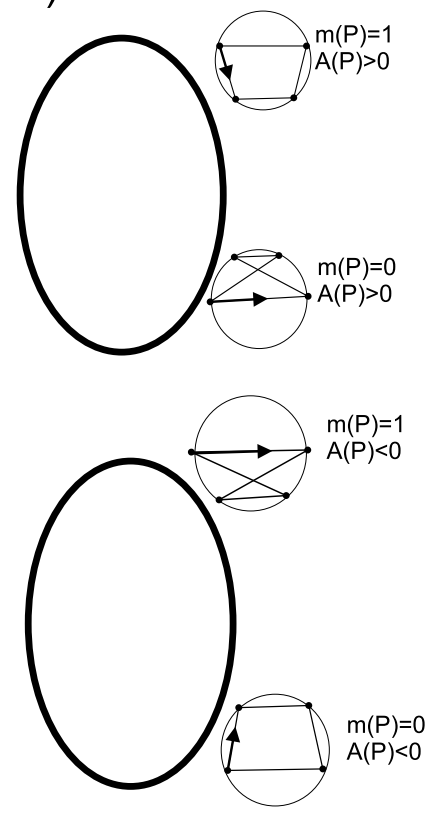

2)

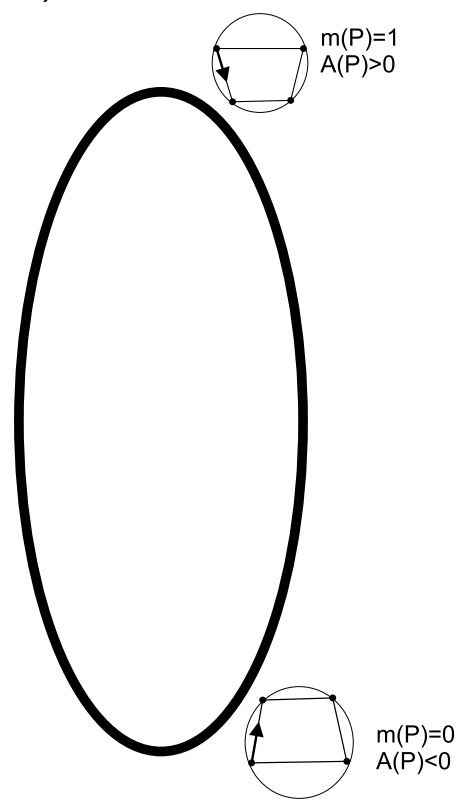

Figure 5. Two types of quadrilateral linkages.

(1) The sum of the lengths of the longest and the shortest edge is less than the sum of the lengths of the other two edges. In this case, the moduli space $\mathcal{M}(L)$ is not connected. It is homeomorphic to a pair of circles and has four cyclic configurations of $L$. They are depicted in Figure 5.1 with their Morse indexes.

(2) The sum of the lengths of the longest and the shortest edge is greater than the sum of the lengths of the other two edges. In this case, the moduli space $\mathcal{M}(L)$ is connected. It is homeomorphic to one circle and has two cyclic configurations of $L$ (Figure 5.2). 


\section{$\S 3$. Morse INDEX}

Theorem 3.1. For a cyclic configuration $P$ of a generic polygonal linkage $L$, we have

$$
m(P)= \begin{cases}e(P)-1-2 \omega(P) & \text { if } \delta(P)>0 \\ e(P)-2-2 \omega(P) & \text { otherwise }\end{cases}
$$

First, we prove the following lemma.

Lemma 3.2. For a generic cyclic n-configuration $P$ such that $e(P)=n$, we have

$$
m(P)=n-1-2 \omega(P) .
$$

Proof. We use induction on the index $\omega(P)$. Since all edges are positively oriented, we have $\omega(P)>0$.

If $\omega(P)=1$, then $P$ is a cyclic convex configuration, which is known to be a point of maximum for $A$. Therefore, $m(P)=n-3$.

For the inductive step, assume that $\omega(P)>1$. Let $k>1$ be the smallest number such that the open chain $\left(p_{1}, p_{2} \ldots \ldots, p_{k}\right)$ intersects itself (Figure 6). The configuration $P$ splits into a homological sum of two configurations

$$
P^{\prime}=\left(p_{1}, p_{2}, \ldots p_{k}\right) \text { and } P^{\prime \prime}=\left(p_{k}, p_{k+1}, \ldots, p_{n}, p_{1}\right)
$$

of two polygonal linkages $L^{\prime}$ and $L^{\prime \prime}$. They are generic by condition 6 .

Since $P^{\prime \prime}$ is positively oriented and

$$
\omega\left(P^{\prime \prime}\right)=\omega(P)-1
$$

we have

$$
m\left(P^{\prime \prime}\right)=n-k-2 \omega(P)+2 .
$$

Now we compute $m\left(P^{\prime}\right)$. We use Theorem 2.8. $P^{\prime}$ has one negatively oriented edge $\left(p_{k}, p_{1}\right)$, and $\left|p_{k}, p_{1}\right|<\left|p_{k-1}, p_{k}\right|$ (see Figure 6). Therefore,

$$
e\left(P^{\prime}\right)=k-1 \text { and } \delta\left(P^{\prime}\right)>0 \text {. }
$$

By Theorem 2.7

$$
\mathcal{H}\left(P^{\prime}\right)=(-1)^{k}
$$

All subconfigurations $P_{i}^{\prime}=\left(p_{1}, \ldots, p_{i}\right)$ of the configuration $P^{\prime}$ for $3 \leq i \leq k-1$ are convex; consequently,

$$
\mathcal{H}\left(P_{i}^{\prime}\right)=(-1)^{i+1} .
$$

Thus, the sequence

$$
\mathcal{H}\left(P_{3}^{\prime}\right), \quad \mathcal{H}\left(P_{4}^{\prime}\right), \quad \mathcal{H}\left(P_{5}^{\prime}\right), \ldots, \quad \mathcal{H}\left(P_{k-1}^{\prime}\right), \quad \mathcal{H}\left(P^{\prime}\right)
$$

looks like this:

$$
1,-1,1,-1,1,-1, \ldots,(-1)^{k},(-1)^{k} .
$$

In other words, we have $k-4$ sign changes (every time except the last). Consequently, $m\left(P^{\prime}\right)=k-4$.

A neighborhood of the point $P^{\prime} \times P^{\prime \prime}$ on the manifold $M_{2}\left(L^{\prime}\right) \times M_{2}\left(L^{\prime \prime}\right)$ admits a natural embedding in a neighborhood of $P$ on the moduli space $M_{2}(L)$ as a codimension one submanifold. Indeed, given a configuration $P_{1}^{\prime}$ of the linkage $L^{\prime}$ and a configuration $P_{1}^{\prime \prime}$ of the linkage $L^{\prime \prime}$, we get a configuration $P_{1}$ by patching them by the edge $\left(p_{1}, p_{k}\right)$.

So, $P_{1}$ is a homological sum of $P_{1}^{\prime}$ and $P_{1}^{\prime \prime}$. We define a function $A$ on the manifold $\mathcal{M}\left(L^{\prime}\right) \times \mathcal{M}\left(L^{\prime \prime}\right)$ as follows:

$$
A\left(P_{1}^{\prime}, P_{1}^{\prime \prime}\right)=A\left(P_{1}^{\prime}\right)+A\left(P_{1}^{\prime \prime}\right)
$$

By Lemma 2.3, this function is the restriction of the signed area function to the manifold $\mathcal{M}(P)$. 


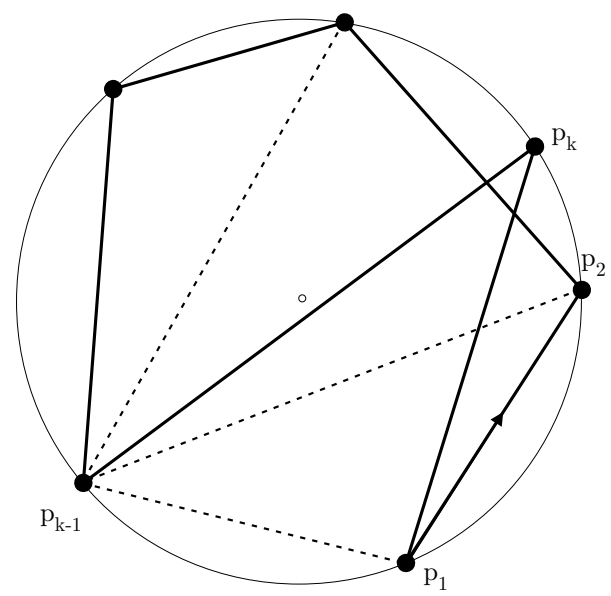

Figure 6 . The configuration $P^{\prime}$.
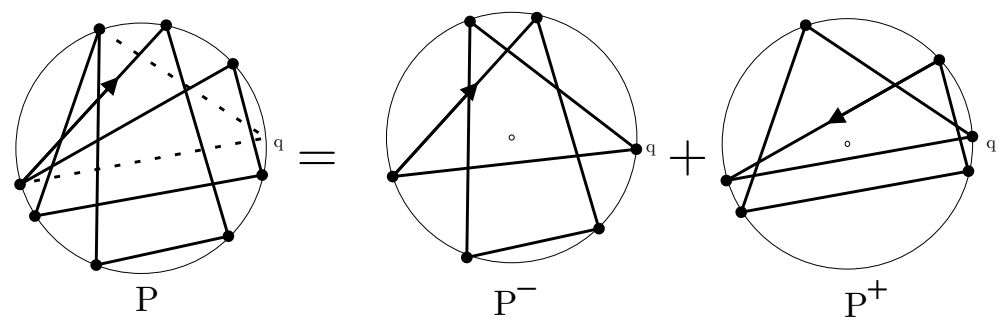

Figure 7. $P$ is a sum of $P^{+}$and $P^{-}$.

Thus, we have a Morse function on a manifold of dimension $n-3$ and its restriction to a submanifold of codimension one. Its Morse indices at a critical point, computed in these two manifolds, differ at most by one.

So, we have either

$$
m(P)=m\left(P^{\prime}\right)+m\left(P^{\prime \prime}\right)=n-1-2 \omega(P),
$$

or

$$
m(P)=m\left(P^{\prime}\right)+m\left(P^{\prime \prime}\right)+1=n-1-2 \omega(P) .
$$

By Theorem 2.7.

$$
\mathcal{H}(P)=d(P)(-1)^{e(P)+1}=(-1)^{n-1} .
$$

That is,

$$
m(P) \equiv n-1 \quad(\bmod 2)
$$

Therefore,

$$
m(P)=n-1-2 \omega(P) .
$$

Lemma 3.3. For a generic cyclic n-configuration $P$ with $e(P)=0$ we have $m(P)=$ $-2 \omega(P)-2$.

Proof. This lemma follows from the previous one for symmetry reasons.

Now we are ready to prove Theorem 3.1 
Proof of Theorem 3.1. By Lemmas 3.2 and 3.3, the claim is true for configurations with $e(P)=0$ or $e(P)=n$.

Consider a cyclic configuration $P$ that has both negatively and positively oriented edges. Assume that for the edges in $P$ we have $\varepsilon_{i}>0$ for $i \leq e(P)$ and $\varepsilon_{i}<0$ for $i>e(P)$. If it is not so, we apply a suitable permutation $\varphi_{\sigma}$ of edges to $P$. By Lemma 2.4, this does not change $m(P)$.

We add a new point $q$ on the circumscribed circle so that the vectors $\overrightarrow{p_{e(P)+1}, \vec{q}}$ and $\overrightarrow{q, p_{1}}$ are positively oriented with respect to $O$. Then $P$ splits into the homological sum of the configuration $P^{+}=\left(p_{1}, p_{2}, \ldots, p_{e(P)+1}, q\right)$ of a linkage $L^{+}$and the configuration $P^{-}=\left(p_{e(P)+1}, \ldots p_{n}, p_{1}, q\right)$ of a linkage $L^{-}$(see Figure 7$)$. The new point can be chosen so that the resulting linkages are generic. The configuration $P^{+}$satisfies the conditions of Lemma 3.2 and $P^{-}$satisfies the conditions of Lemma 3.3. Therefore,

$$
m\left(P^{+}\right)=e(P)-2 \omega\left(P^{+}\right)+1
$$

and

$$
m\left(P^{-}\right)=-2 \omega\left(P^{-}\right)-2 .
$$

A neighborhood of $P$ in the manifold $M_{2}(L)$ admits a natural embedding in a neighborhood of $P^{+} \times P^{-}$in the manifold $M_{2}\left(L^{+}\right) \times M_{2}\left(L^{-}\right)$of codimension one. Therefore, either

$$
m(P)=m\left(P^{+}\right)+m\left(P^{-}\right)=e(P)-1-2 \omega(P),
$$

or

$$
m(P)=m\left(P^{+}\right)+m\left(P^{-}\right)-1=e(P)-2-2 \omega(P) .
$$

Two cases are possible:

(1) $\delta(P)>0$. Then, by Theorem 2.7 $m(P) \equiv e(P)+1(\bmod 2)$. Therefore, $m(P)=$ $e(P)-1-2 \omega(P)$.

(2) $\delta(P)<0$. Then, by Theorem 2.7, $m(P) \equiv e(P)(\bmod 2)$. Therefore, $m(P)=$ $e(P)-2-2 \omega(P)$.

\section{$\S 4$. LOCAL MAXIMA OF THE SIGNED AREA FUNCTION}

With the use of Theorem 3.1, we can get a full description of the local maxima for the signed area function. It is reasonable to distinguish four types of maxima. Criteria for all except for the last type are purely combinatorial (no condition on $\delta(P)$ ), while the fourth case involves the condition $\delta(P)>0$.

Theorem 4.1. Let $P$ be a cyclic configuration of a generic polygonal linkage.

We assume that $\varepsilon_{i}=1,1 \leq i \leq e(P)$. If this is not the case, then we apply a suitable permutation $\varphi_{\sigma}$ of sides (see Lemma 2.4) such that this condition is fulfilled in $\varphi_{\sigma}(P)$. The point $P$ is a point of a local maximum of the signed area function $A$ if and only if $P$ is a configuration of one of the following four types.

(1) $P$ is a convex configuration (Figure 8.1).

(2) $P$ consists only of negatively oriented edges and every two edges cross each other (Figure 8.2).

(3) The following three conditions are satisfied (Figure 8.3):

- every two negatively oriented edges cross each other;

- the positively oriented edges do not cross each other;

- no positively oriented edge crosses a negatively oriented edge. 

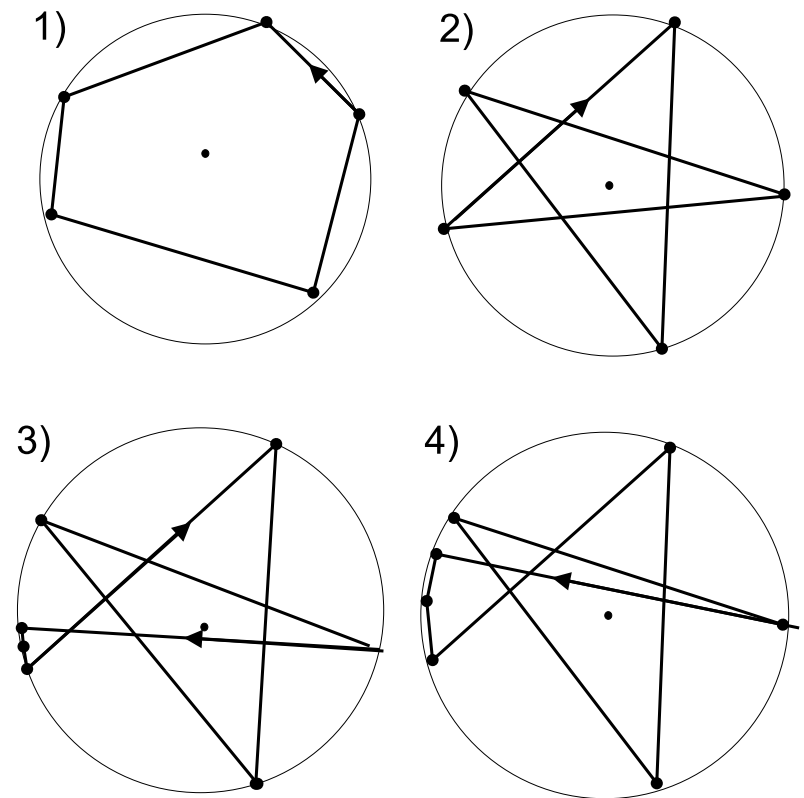

Figure 8. Four types of local maxima of A.

(4) The following four conditions are satisfied (Figure 8.4):

- $\delta(P)>0$;

- every two negatively oriented edges cross each other;

- the positively oriented edges do not cross each other;

- only one positively oriented edge crosses negatively oriented edges, and it crosses them all.

Remark 4.2. Talking of the edges of the configuration that cross or do not cross each other, we mean all pairs of edges that are not adjacent.

First, we prove the following lemma.

Lemma 4.3. If a cyclic configuration is a point of local maximum for $A$, then every two its negatively oriented edges cross each other.

To prove this, we need the notion of bending of a linkage.

Definition 4.4. Let $L$ be a linkage. We define its bending as a continuous mapping of an interval of the real line into the moduli space $\mathcal{M}(L)$.

Proof of Lemma 4.3. Suppose the contrary, i.e., suppose that a configuration $P$ is a maximum and two its negatively oriented edges not cross each other. Let them be the edges $\left(p_{k}, p_{k+1}\right)$ and $\left(p_{l}, p_{l+1}\right), k<l$. Consider the quadrilateral subconfiguration $Q=\left(p_{k}, p_{k+1}, p_{l}, p_{l+1}\right)$. It can be either an anticonvex configuration, or a self-crossing configuration with positive area (see Figure 5). Both of them are local minima (see Example 2.9), and all their bendings increase their area. If we fix the subconfigurations $\left(p_{1}, p_{2}, \ldots, p_{k}, p_{l+1}, \ldots, p_{n}\right)$ and $\left(p_{k+1}, p_{k+2}, \ldots, p_{l}\right)$, then for every bending $Q(t)$ of $Q$ we shall have a bending $P(t)$ of $P$ such that

$$
A(P(t))=A(Q(t))+\text { const. }
$$

So, there is a bending that increases the area of $P$; consequently, $P$ is not a maximum. 
The following lemma is proved similarly.

Lemma 4.5. If a cyclic configuration is a point of local maximum for A, then every two positively oriented edges do not cross each other.

Proof of Theorem 4.1. First, we prove that configurations of all four types are local maxima.

(1) Obvious.

(2) Count the winding number $\omega(P)$ of the curve $P$ with respect to the point $O$. Recall that $\omega(P)$ is equal to the number of intersections (with sign) of the curve $P$ with any ray that comes out of $O$. We use the ray $\left(O, p_{2}\right)$.

All edges except $\left(p_{1}, p_{2}\right),\left(p_{2}, p_{3}\right)$, and $\left(p_{n}, p_{1}\right)$ cross the edge $\left(p_{1}, p_{2}\right)$. Half of these $n-3$ edges has a starting point on the smaller of the two $\operatorname{arcs}\left(p_{1}, p_{2}\right)$, and the other half has the endpoint there. Every edge that starts on this arc crosses the ray $\left(O, p_{2}\right)$ in the negative direction. The configuration $P$ crosses this ray at one more point, namely, at $p_{2}$, also in the negative direction.

Thus, we have:

$$
\omega(P)=-\left(\frac{n-3}{2}+1\right)=\frac{-n+1}{2} .
$$

We know that $e(P)=0$. Therefore, $\delta(P)<0$. By Theorem 3.1

$$
m(P)=0-(-n+1)-2=n-3 .
$$

That is, $P$ is a point of local maximum for the function $A$.

(3) Again, we count $\omega(P)$. For this, consider the number and the signs of the intersections of the configuration in question and the ray $\left(O, p_{e(P)+2}\right)$. All negatively oriented edges except $\left(p_{e(P)+1}, p_{e(P)+2}\right)$ and $\left(p_{e(P)+2}, p_{e(P)+3}\right)$ cross the edge $\left(p_{e(P)+1}, p_{e(P)+2}\right)$. Since no positively oriented edge crosses $\left(p_{e(P)+1}, p_{e(P)+2}\right)$, and all edges that connect the vertices $p_{1}$ and $p_{e(P)+1}$ are positively oriented, the point $p_{1}$ lies on the smaller of the $\operatorname{arcs}\left(p_{e(P)+1}, p_{e(P)+2}\right)$. Consequently, the negatively oriented edge $\left(p_{n}, p_{1}\right)$ does not cross the ray $\left(O, p_{e(P)+2}\right)$. The remaining negatively oriented edges cross the edge $\left(p_{e(P)+1}, p_{e(P)+2}\right)$. Half of them has a starting point on this arc, and therefore, crosses the ray $\left(O, p_{e(P)+2}\right)$ in the negative direction.

The configuration $P$ also crosses this ray at the point $p_{e(P)+2}$ in the negative direction.

Consequently,

$$
\omega(P)=\frac{-n+e(P)+1}{2} .
$$

Since $\alpha_{n}>\alpha_{1}+\alpha_{2}+\cdots+\alpha_{e(P)}$, and for $0<\alpha<\frac{\pi}{2}$ the function $\tan \alpha$ is convex, we have (Figure 8.3)

Hence, $\delta(P)<0$.

$$
\tan \alpha_{n}>\tan \alpha_{1}+\tan \alpha_{2}+\cdots+\tan \alpha_{e(P)} .
$$

So, by Theorem 3.1, we have

$$
m(P)=e(P)-(-n+e(P)+1)-2=n-3 .
$$

That is, $P$ is a point of local maximum for the function $A$.

(4) Again, we consider the number and the signs of the intersections of $P$ and the ray $\left(O, p_{e(P)+2}\right)$.

Only one positively oriented edge of $P$ crosses the edge $\left(p_{e(P)+1}, p_{e(P)+2}\right)$ that is negatively oriented. Let it be $\left(p_{k}, p_{k+1}\right), 1 \leq k \leq e(P)$.

The vertices $p_{k+1}$ and $p_{e(P)+1}$ are connected by a chain of positively oriented edges none of which crosses the edge $\left(p_{e(P)+1}, p_{e(P)+2}\right)$. Therefore, the vertex $p_{k+1}$ lies on the smaller of the two arcs $\left(p_{e(P)+1}, p_{e(P)+2}\right)$. So, the positively oriented edge $\left(p_{k}, p_{k+1}\right)$ crosses the ray $\left(O, p_{e(P)+2}\right)$ in the positive direction. 
All negatively oriented edges except $\left(p_{e(P)+1}, p_{e(P)+2}\right)$ and $\left(p_{e(P)+2}, p_{e(P)+3}\right)$ cross the edge $\left(p_{e(P)+1}, p_{e(P)+2}\right)$. Half of them have the starting point on the smaller of the $\operatorname{arcs}\left(p_{e(P)+1}, p_{e(P)+2}\right)$, which means that they cross the ray $\left(O, p_{e(P)+2}\right)$ in the negative direction.

Moreover, the configuration $P$ crosses the ray $\left(O, p_{e(P)+2}\right)$ at the point $p_{e(P)+2}$ in the negative direction.

Consequently,

$$
\omega(P)=-\frac{n-e(P)-2}{2}+1-1=-\frac{n-e(P)-2}{2} .
$$

Since $\delta(P)>0$, by Theorem 3.1 we have

$$
m(P)=e(P)+2 \frac{n-e(P)-2}{2}-1=n-3 .
$$

That is, the configuration $P$ is a local maximum of $A$.

Now we prove that every local maximum belongs to one of the four types listed above. Let configuration $P$ be a local maximum, i.e., let $m(P)=n-3$.

We consider three possible cases.

(1) $e(P)=n$ (all edges are positively oriented).

Then $\delta(P)>0$ and, by Theorem 3.1, $\omega(P)=1$, i.e., $P$ is a convex configuration and belongs to type 1 .

(2) $e(P)=0$ (all edges are negatively oriented).

Then, by Lemma 4.3. every two edges of the configuration $P$ cross each other, and the configuration belongs to type 2 .

(3) $0<e(P)<n$.

Split $P$ to a homological sum of two subconfigurations $P^{+}$and $P^{-}$, as we did in the proof of Theorem 3.1. Note that

$$
\omega(P)=\omega\left(P^{-}\right)+\omega\left(P^{+}\right) .
$$

Two subcases are possible.

Subcase 1: $\delta(P)<0$. By Theorem 3.1, we have

$$
\omega(P)=\frac{-n+e(P)+1}{2} .
$$

Since $P^{-}$consists of $n-e(P)+2$ edges, we can write

$$
\omega\left(P^{-}\right)>\frac{-n+e(P)-2}{2}=\omega(P)-\frac{3}{2} .
$$

Note that $\omega\left(P^{+}\right) \geq 1$, so that $\omega\left(P^{-}\right)<\omega(P)$. This, we have

$$
\omega\left(P^{-}\right)=\omega(P)-1=\frac{-n+e(P)-1}{2}
$$

and

$$
\omega\left(P^{+}\right)=1 .
$$

Consequently, the number of negatively oriented edges in $P$, which equals $n-e(P)$, is odd.

Since, by Lemma 4.3, all negatively oriented edges cross each other, all vertices $p_{e(P)+2 k}$, from $p_{e(P)+4}$ and to $p_{n+1}$ (recall that $p_{n+1}=p_{1}$ ), lie on the smaller of the two $\operatorname{arcs}\left(p_{e(P)+1}, p_{e(P)+2}\right)$, and all the vertices $p_{e(P)+2 k-1}$ from $p_{e(P)+3}$ to $p_{n}$ lie on the larger arc $\left(p_{e(P)+1}, p_{e(P)+2}\right)$.

We prove that the above two groups of vertices are ordered by their numbers clockwise.

Indeed, let $i$ be the smallest number of a vertex that breaks this order. Then the vector $\overrightarrow{p_{i-2}, p_{i}}$ is negatively oriented with respect to $O$. Since the edges $\left(p_{i-2}, p_{i-1}\right)$ and 
$\left(p_{i-1}, p_{i}\right)$ are negatively oriented, the vertex $p_{i+1}$ lies on the smaller of the $\operatorname{arcs}\left(p_{i-2}, p_{i}\right)$. Since the vertices $p_{i-2}$ and $p_{i}$ lie on one and the same side of $\left(p_{e(P)+1}, p_{e(P)+2}\right)$, the edge $\left(p_{i-1}, p_{i}\right)$ does not cross $\left(p_{e(P)+1}, p_{e(P)+2}\right)$, a contradiction.

From the above, it follows that there are no vertices of negatively oriented edges on the smaller arc $\left(p_{1}, p_{e(P)+1}\right)$. Recall that, by Lemma 4.5, the positively oriented edges do not cross each other. So, the sum of all smaller arcs that correspond to positively oriented edges is less than $2 \pi$. All positively oriented edges form a chain connecting $p_{1}$ and $p_{e(P)+1}$. There are no vertices of negatively oriented edges between $p_{1}$ and $p_{e(P)+1}$ in the positive direction. So, the positively oriented edges do not cross negatively oriented edges, which implies that the configuration belongs to type 3 .

Subcase 2: $\delta(P)>0$. By Theorem 3.1, we have

$$
\omega(P)=\frac{-n+e(P)+2}{2} .
$$

Since $P^{-}$consists of $n-e(P)+2$ edges, we can write

$$
\omega\left(P^{-}\right)>\frac{-n+e(P)-2}{2}=\omega(P)-2 .
$$

Note that $\omega\left(P^{+}\right) \geq 1$ whence $\omega\left(P^{-}\right)<\omega(P)$. So, we have

$$
\omega\left(P^{-}\right)=\omega(P)-1=\frac{-n+e(P)}{2}
$$

and

$$
\omega\left(P^{+}\right)=1 .
$$

Therefore the number of negatively oriented edges in $P$, which equals $n-e(P)$, is even. As in the first subcase, all vertices $p_{e(P)+2 k+1}$ incident to negatively oriented edges lie on the larger of the arcs $\left(p_{e(P)+1}, p_{e(P)+2}\right)$, and the vertices $p_{e(P)+2 k}$ incident to negatively oriented edges lie on the smaller of these arcs, and both groups are ordered clockwise. But in this case the vertex $p_{1}=p_{n+1}$ lies on the larger arc.

The vertices $p_{1}$ and $p_{e(P)+1}$ are connected by the chain of all positively oriented edges. Since only the vertices $p_{e(P)+2 k}$ lie on the circle between $p_{1}$ and $p_{e(P)+1}$ in the positive direction, the positively oriented edges must cross all negatively oriented edges incident to the vertices $p_{e(P)+2 k}$, that is, all negatively oriented edges.

Now we prove that there is only one positively oriented edge that crosses the negatively oriented edges. Suppose the contrary, i.e., at least two positively oriented edges cross the negatively oriented edges. Recall that the positively oriented edges form a chain connecting the vertices $p_{1}$ and $p_{e(P)+1}$. Also, recall that the vertices $p_{e(P)+2 k}$ incident to negatively oriented edges lie on the positively oriented arc between $p_{1}$ and $p_{e(P)+1}$. Among them, those nearest to the ends are $p_{n}$ (the nearest to $\left.p_{e(P)+1}\right)$ and $p_{e(P)+2}$ (the nearest to $p_{1}$ ). If at least two positively oriented edges cross the negatively oriented edges, then the vertices $p_{n}$ and $p_{e(P)+2}$ must lie on arcs corresponding to different positively oriented edges (see Figure 9 ). So, at least one vertex on the arc between them is incident to a positively oriented edge. Let it be the vertex $p_{l}$.

Denote by $\alpha^{\prime}$ and $\alpha^{\prime \prime}$ the halves of the central angles corresponding to $\left(p_{1}, p_{l}\right)$ and $\left(p_{l}, p_{e(P)+1}\right)$. Note that, since $\left|\left(p_{1}, p_{l}\right)\right|<l_{n}$ and $\left|\left(p_{l}, p_{e(P)+1}\right)\right|<l_{e(P)+1}$ (see Figure 9$)$, we have

$$
\tan \alpha^{\prime}+\tan \alpha^{\prime \prime}<\tan \alpha_{n}+\tan \alpha_{e(P)+1}
$$




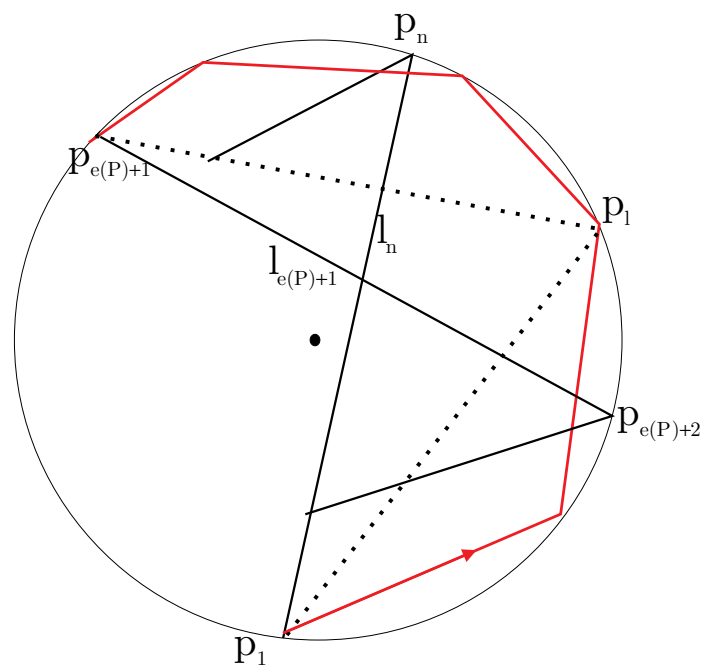

FiguRE 9. This configuration is not a maximum.

Also, since

$$
\begin{aligned}
\alpha_{1}+\alpha_{2}+\cdots+\alpha_{l-1} & =\alpha^{\prime}<\frac{\pi}{2}, \\
\alpha_{l}+\alpha_{l+1}+\cdots+\alpha_{e(P)} & =\alpha^{\prime \prime}<\frac{\pi}{2}
\end{aligned}
$$

and for $0<\alpha<\frac{\pi}{2}$ the function $\tan \alpha$ is convex, we have

$$
\tan \alpha_{1}+\tan \alpha_{2}+\cdots+\tan \alpha_{e(P)}<\tan \alpha^{\prime}+\tan \alpha^{\prime \prime} .
$$

Consequently,

$$
\tan \alpha_{e(P)+1}+\tan \alpha_{n}<\tan \alpha_{e(P)+1}+\tan \alpha_{e(P)+2}+\cdots+\tan \alpha_{n} .
$$

Therefore, the sum of the tangents of the angles corresponding to the positively oriented edges is less than the sum of the tangents of the angles corresponding to the negatively oriented edges. Therefore, $\delta(P)<0$, a contradiction.

Thus, there is only one positively oriented edge that crosses the negatively oriented edges. Moreover, by Lemma 4.5, the positively oriented edges do not cross each other. The configuration belongs to type 4 .

By symmetry reasons, from Theorem 4.1 we get the following corollary.

Corollary 4.6. Let $P$ be a cyclic configuration of a generic polygonal linkage.

Assume that $\varepsilon_{i}=1,1 \leq i \leq e(P)$. If it is not so, we apply a suitable permutation $\varphi_{\sigma}$ of sides (see Lemma 2.4) such that this condition is fulfilled in $\varphi_{\sigma}(P)$. The point $P$ is a point of local minimum of the signed area function $A$ if and only if $P$ is a configuration of one of the following four types.

(1) $P$ is an anticonvex configuration (Figure 8.1).

(2) $P$ has only positively oriented edges and every two edges cross each other (Figure 8.2).

(3) The following three conditions are satisfied (Figure 8.3):

- every two positively oriented edges cross each other;

- the negatively oriented edges do not cross each other;

- no negatively oriented edge crosses a positively oriented edge. 
(4) The following four conditions are satisfied (Figure 8.4):

- $\delta(P)<0$;

- every two positively oriented edges cross each other;

- the negatively oriented edges do not cross each other;

- only one negatively oriented edge crosses positively oriented edges, and it crosses them all.

\section{REFERENCES}

[1] J. Cerf, La stratification naturelle des espaces de fonctions différentiables réelles et le théorème de la pseudo-isotopie, Inst. Hautes Études Sci. Publ. Math., No. 39 (1970), 5-173. MR0292089 (45:1176)

[2] E. Elerdashvili, M. Jibladze, and G. Khimshiashvili, Cyclic configurations of pentagon linkages, Bull. Georgian Natl. Acad. Sci. 2 (2008), no. 4, 29-32. MR2555487

[3] M. Farber and D. Schütz, Homology of planar polygon spaces, Geom. Dedicata 125 (2007), 75-92. MR2322541(2008c:58007)

[4] C. Gibson and P. Newstead, On the geometry of the planar 4-bar mechanism, Acta Appl. Math. 7 (1986), 113-135. MR860287 (88a:14037)

[5] G. Khimshiashvili, On configuration spaces of planar pentagons, Zap. Nauchn. Sem. S.-Peterburg. Otdel. Mat. Inst. Steklov. (POMI) 292 (2002), 120-129; English transl., J. Math. Sci. (N.Y.) 126 (2005), no. 2, 1111-1116. MR1944087 (2003k:55019)

[6] , Configuration spaces and signature formulae, Algebra and Geometry, J. Math. Sci. (N.Y.), 160 (2009), no. 6, 727-736. MR2760304 (2012e:55019)

[7] G. Khimshiashvili and G. Panina, Cyclic polygons are critical points of area, Zap. Nauchn. Sem. S.-Peterburg. Otdel. Mat. Inst. Steklov. (POMI) 360 (2008), 238-245; English transl., J. Math. Sci. (N.Y.) 158 (2009), no. 6, 899-903. MR2759748(2012d:52033)

[8] G. Panina and A. Zhukova, Morse index of a cyclic polygon, Cent. Eur. J. Math. 9 (2011), no. 2, 364-377. MR2772432 (2012d:52024)

[9] G. Polya, Induction and analogy in mathematics, Mathematics and Plausible Reasoning, vol. 1, Princeton Univ. Press, Princeton, NJ, 1954. MR0066321 (16:556f)

[10] D. Robbins, Areas of polygons inscribed in a circle, Discrete Comput. Geom. 12 (1994), no. 2, 223-236. MR 1283889 (95g:51027)

[11] D. Zvonkine, Configuration spaces of hinge constructions, Russian J. Math. Phys. 5 (1997), no. 20, 247-266. MR1491636 (99c:52034)

Department of Mathematics and Mechanics, St. Petersburg State University, Petrodvorets, St. Petersburg 198904, Russia

E-mail address: millionnaya13@ya.ru

Received 29/MAY/2011

Translated by THE AUTHOR 